\title{
The Galactic Evolution of Boron
}

\author{
Francesca Primas \\ European Southern Observatory, Karl- Schwarzschildstr. 2, D-85748 \\ Garching bei München
}

\begin{abstract}
Boron, together with lithium and beryllium, belongs to the group of the so-called light elements, the importance of which ranges from providing important tests to Big Bang nucleosynthesis scenarios to being useful probes of stellar interiors and useful tools to further constrain the chemical evolution of the Galaxy.

Since it became operative in the late eighties, the Hubble Space Telescope (HST) and its high- and medium-resolution spectrographs have played a key role in analyzing boron. Boron has now been observed in several stars and in the interstellar medium (ISM), providing important information in different fields of astrophysical research (nucleosynthesis, cosmic-ray spallation, stellar structure). In particular, determinations of boron in unevolved stars of different metallicity have allowed to study how boron evolves with iron.

After a general review of the current status of boron observations and of the major uncertainties affecting the measurements of its abundance, I will mainly concentrate on unevolved stars and discuss the "evolutionary" picture emerging from the most recent analyses and how its interpretation compares with theoretical expectations. A brief discussion on future prospects will conclude this contribution, showing how the field may evolve and improve.
\end{abstract}

\section{Introduction}

Abundances of the light elements $\mathrm{Li}, \mathrm{Be}$, and $\mathrm{B}$ play a critical role in understanding stellar mixing, Big-Bang nucleosynthesis (BBN), and galactic chemical evolution. The stellar structure interest (which will not be discussed here) stems from the fact that all three elements cannot survive in deep stellar interiors because they burn at progressively higher temperatures $\left(\sim 2.5,3.5\right.$ and $\left.5.0 \times 10^{6} \mathrm{~K}\right)$ at densities found in $\mathrm{F}$ and $\mathrm{G}$ stars near the base of the surface convection zone. Therefore circulation and destruction of the light elements can result in observable abundance changes, which become a powerful diagnostic for testing stellar structure models and internal mixing.

The "cosmological" interest, on the other hand, derives from the fact that the analysis of boron abundances as well as of the other two light nuclides in different types of stellar objects represents an additional test of the standard Big Bang nucleosynthesis theory, and can further constrain the galactic cosmicray (GCR) spallation scenario, responsible for Be and $B$ formation. It is well 
known that standard Big Bang nucleosynthesis predicts primordial production of ${ }^{7} \mathrm{Li}$, but not of ${ }^{6} \mathrm{Li},{ }^{9} \mathrm{Be},{ }^{10} \mathrm{~B}$, and ${ }^{11} \mathrm{~B}$, which have long been believed to arise from spallation reactions involving protons and/or $\alpha$-particles colliding with the nuclei of the abundant elements carbon, nitrogen, and oxygen (CNO). The abundance from this source is expected to build up from near zero at the time of the formation of the Galaxy to the values observed in the interstellar medium today, at a rate which depends on the high energy particle flux and the abundance of the target nuclei CNO. From the analysis of light element abundances in stars of different metallicity it is possible to follow their galactic evolution and constrain which mechanisms are responsible for their production. This can be inferred, for instance, by investigating the slope of the trend $\mathrm{Be}, \mathrm{B}$ versus $\mathrm{Fe}$ or by testing the presence of a knee in this trend and, if any, correlating it with plausible dynamical events in the formation of the halo according to the metallicity at which such change of slope is found. Until quite recently, however, there was very little information about the evolution of these elements throughout the history of the Galaxy. The prediction of a possible primordial production of $\mathrm{Be}$ and $\mathrm{B}$ in some inhomogeneous Big Bang models (cf Orito et al. 1997, and references therein) has helped in keeping alive the interest in how $\mathrm{Be}$ and $\mathrm{B}$ behave especially at the lowest metallicities alive. In other words, if a plateau were to be detected similarly to the one found in the case of lithium (the "Spite-plateau", cf Spite \& Spite 1982).

As originally proposed by Reeves, Fowler, \& Hoyle (1970), the rate of formation of ${ }^{9} \mathrm{Be}$ (as well as of ${ }^{10} \mathrm{~B}$ ) in GCR reactions is given by the product of the flux of high-energy protons times the cross sections for ${ }^{9} \mathrm{Be}$ formation by proton collision on the most abundant targets, ${ }^{16} \mathrm{O}$ and ${ }^{12} \mathrm{C}$, times the abundance ratio of these targets with respect to hydrogen in space, i.e. :

$$
d N_{B e} / d t \propto \chi(t) \times \int \sigma(E) \times \Phi(E, t) d E-\text { loss terms }
$$

where $\chi(\mathrm{t})$ is the relative abundance of the target heavy elements, $\sigma(\mathrm{E})$ the reaction cross section (i.e. measured in a laboratory), which depend on the energy $E$ of the cosmic rays, and $\Phi(E, t)$ the time-dependent cosmic-ray flux.

Based on the data available at that time, the approximate equality found between the product of the formation rate times the age of the Galaxy, on one hand, and the beryllium-to-hydrogen ratio measured in young stars $\left(\sim 10^{-11}\right)$ was interpreted as a strong evidence for a major GCR contribution to some of the light elements. Indeed, the comparison of the ratios of the spallation cross sections of protons on $\mathrm{O}$ and $\mathrm{C}$ to the ratios of the stellar abundances of $\mathrm{Li}, \mathrm{Be}$ and $B$ shows that the GCR mechanism can satisfactorily account for the nuclei ${ }^{6} \mathrm{Li},{ }^{9} \mathrm{Be}$, and ${ }^{10} \mathrm{~B}$. Cosmic-ray spallation in the general ISM has therefore been accepted for most of the past 25 years as the main site of production of these light isotopes, with the only exception of ${ }^{7} \mathrm{Li}\left(10 \%\right.$ contribution) and ${ }^{11} \mathrm{~B}$ (likely contribution from $\nu$-spallation).

The build-up of Be and B in the Galaxy thus depends on the CR density, which presumably depends on the supernova rate and, in turn, on the star formation rate. The yields also depend on the rise of the (progenitor) $\mathrm{CNO}$ abundances and the decline of the gas mass fraction. Furthermore, the cumulative abundances are affected by the rates of infall of fresh (unprocessed) material and outflow, e.g. from supernova heating, and the time of formation of 
the halo and the disk. In such scenario, the light nuclide (boron, for instance) is expected to follow oxygen with a quadratic slope. In fact, since the primary process of spallation involves cosmic-ray protons and $\alpha$-particles on interstellar heavy nuclei (mostly $\mathrm{O}$ ), the increase of $\mathrm{B}$ is related to the supernova rate and the current $\mathrm{O}$ abundance. Being also $\mathrm{O}$ dependent on the supernova rate, the resulting dependence is quadratic.

\section{Boron Observations}

There are not many transitions available to measure boron abundances, and most of them fall in the ultraviolet part of the spectrum, thus requiring spacebased observing facilities. This clearly explains why the Hubble Space Telescope had such a strong impact in the past decade on this specific field of research. The transitions most widely used for abundance measurements in stellar atmospheres are the resonance doublet of B I at $2497 \AA$ (typically used in cool stars, e.g. Duncan, Lambert, \& Lemke 1993; Duncan et al. 1997; Primas et al. 1998, Primas et al. 1999) and the resonance line of B II at $1362 \AA$ (preferentially used in early-type stars, e.g. Venn, Lambert, \& Lemke 1996). Moreover, Johansson et al. (1993) showed that the most favourable transition to attempt a determination of the isotopic ratio ${ }^{11} \mathrm{~B} /{ }^{10} \mathrm{~B}$ is the $\mathrm{B} \mathrm{I}$ line at $2090 \AA$, for which they predict an isotopic shift of $25 \mathrm{~m} \AA$ (to be compared to the $8 \mathrm{~m} \AA$ only in the case of the B I line at $2497 \AA$ ). Rebull et al. (1999) have reported on their first attempt in such direction. Finally, at this symposium, Meléndez \& Barbuy (2000) have shown that it may be possible to study boron in cool giants and supergiants via the infrared B I transitions at 1.166 and $1.624 \mu$.

\subsection{Historical Background and Current Observational Status}

Although the Hubble Space Telescope marked a new era of boron observations, there have been several analyses of boron before it was launched. One of the first studies of boron to appear in an official scientific journal is the work by Rowland \& Tatnall (1895), "The Arc-spectra of the Elements.I. Boron and Beryllium" (in the first volume of the Astrophysical Journal). The first attempts of detecting boron in stellar atmospheres were of course devoted to the brightest and closest stars like the Sun (e.g. Nicholson \& Perrakis 1928), and were then followed by more detailed analyses: in the Sun (e.g. Kohl, Parkinson, \& Withbroe 1977), in Sirius (Praderie et al. 1977), and for the first time in A- and B-type stars (Boesgaard \& Heacox 1978). The eighties were characterized by the International Ultraviolet Explorer and the first attempts to determine boron in metal-poor stars (Molaro 1987), whereas the Hubble Space Telescope clearly dominated the last decade of research. Thanks to this remarkable technological improvement, it finally became possible to start the first systematic analyses of boron. Duncan et al. (1992) took advantage of the first cycle of HST observations to show that the three metal-poor dwarf stars analyzed by them showed a surprising linear growth of boron abundances with increasing metallicity. A finding further confirmed by later analyses that included larger samples of data points (cf Duncan et al. 1997, Primas et al. 1999).

After 8 years of HST operations, the current status of boron observations includes, a part from the Sun and some other bright objects, a relevant number of 
early type galactic and extragalactic stars (cf Venn et al. 1996, and Cunha et al. 1997 respectively), galactic F- and G-type stars (cf Duncan et al. 1997; Primas et al 1999; García-López et al. 1999; Boesgaard et al. 1999), and different lines of sight in local diffuse interstellar clouds. Lambert et al. (1998), for instance, measured the boron isotopic ratio to be $3.4 \pm 0.7$ dex in 3 different lines of sight (towards $\zeta \mathrm{Oph}, \kappa$ Ori and $\delta \mathrm{Sco}$ ).

\subsection{Difficulties Associated to Boron Measurements}

Different factors affect the final accuracy of each boron measurement. In the first place, the quality of the observed data. All of the data available today for old galactic stars have been derived from the resonant doublet at $2500 \AA$ (mainly from the bluer of the 2 lines, the redder one being blended), hence they were collected with the Hubble Space Telescope. The choice of gratings is then limited to high or medium resolution, but very few spectra have been observed with the high-resolution echelle grating characterized by a nominal resolving power $\mathrm{R} \sim 90,000$ (with the Goddard High Resolution Spectrograph). Edvardsson et al. (1993) analyzed one of the well-known very metal-poor stars, HD 140283 $([\mathrm{Fe} / \mathrm{H}]=-2.60)$, with this instrumental set-up, and remarkably constrained the presence and strength of blending lines. But because this is a very time consuming choice, most of the boron data that will be shown and discussed here have been observed at $R \sim 30,000$. Figure 1 shows the spectral region around $2500 \AA$ of an intermediate metallicity star (HD 94028, $[\mathrm{Fe} / \mathrm{H}] \sim-1.5)$. The continuous line represent the best fit to the data (here represented by photon statistics errorbars), whereas the dot-dashed lines were computed with \pm 0.20 dex change in boron abundance. The dotted line is the synthesis calculated without boron.

The second group of uncertainties is more related to the method of analysis. One of the main requirements for a proper analysis of the UV spectral region is the need of spectrum synthesis (cf Fig. 1). The crowding is severe and a high percentage of absorption features is blended. Such technique requires three main inputs: a reliable list of atomic and molecular lines, a grid of model atmospheres, and the determination of the main stellar parameters of the objects under investigation, i.e. effective temperature, gravity, and metallicity. Each of these items introduces different uncertainties, which must be carefully taken into account in the final computation of the uncertainties. On one side, one must include the uncertainties coming from laboratory measurements of the wavelengths and the oscillator strengths of each atomic and molecular transition, the physics behind the formation of the boron lines (and others) in the stellar atmosphere (Local Thermodynamic Equilibrium versus Non-Local Thermodynamic Equilibrium), and on the other hand one should keep in mind that current model atmospheres probably do not give a realistic description of a stellar atmosphere (1-dimensional versus 3-dimensional). Moreover, the effect and importance of possible blendings (like the Co I line at $2496.708 \AA$ very close to the bluer B I line at $2496.772 \AA$ ) should also be quantified.

Despite this scenario may look quite discouraging, boron analyses carried out during the last few years have indeed introduced several improvements. Boron lines form under NLTE conditions (cf Kiselman \& Carlsson 1994), hence corrections to the abundances usually determined under the simpler assumption of LTE must be (and have been) applied. The development of 3-D hydrody- 


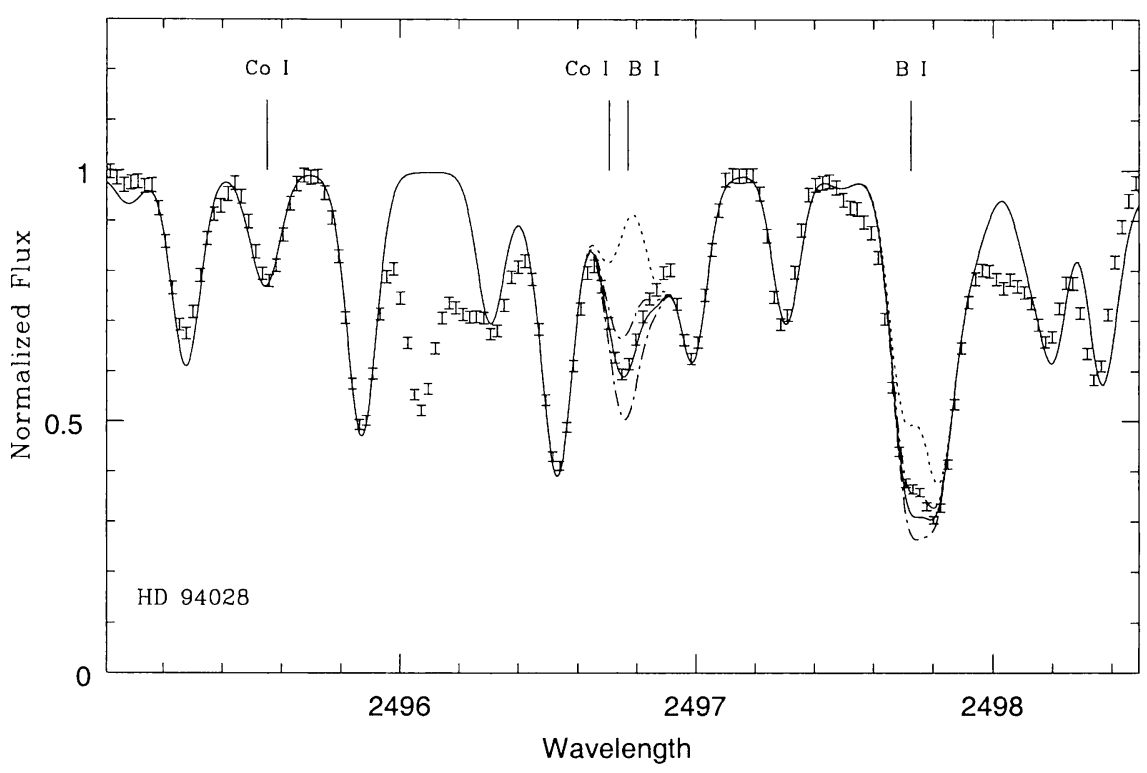

Figure 1. A portion of the spectrum of HD 94028 (cf Primas et al. 1999) around the B I resonance doublet. See text for more explanations.

namical models is in progress (cf Asplund et al. 1999; Asplund 2000): this will represent a remarkable step forward. As far as the problem of blendings is concerned, Peterson, Carney, \& Smith (1999) suggested an improved solution for the treatment of the most important blend affecting B measurements: the neutral cobalt line at $\lambda 2496.708$. By taking advantage of the "unblended" (clean) Co I line at $2495.55 \AA$, they were able to much better constain the cobalt abundance. Such an approach was readily implemented in the most recent analysis by Primas et al. (1999). Once all these different sources of uncertainty have been taken into account, typical error bars usually range between 0.10 and 0.25 dex.

Finally, it is important to notice that for the interpretation of the evolutionary trend of boron abundances, the size of the available data sample plays an important role too. Among the 3 light elements, boron abundances are indeed the least explored, mainly because its UV lines can be observed with space-based facilities only.

\section{Observational Results}

Because the increase of $\mathrm{B}$ and $\mathrm{Be}$ data during the past few years has been indeed remarkable (compared to 10 years ago, for instance), the study of $\mathrm{Li}, \mathrm{Be}$, and $\mathrm{B}$ evolutionary trends have become to be regarded as a powerful discriminant between different models of the chemical and dynamical evolution of the Galaxy. 
The evolution of $B$ has received considerable attention, since observations of its abundance in halo stars have shown that:

a) its relationship with respect to iron is a linear one, over 3 decades of metallicity;

b) it evolves with a constant ratio of $\mathrm{B} / \mathrm{Be} \sim 10-20$, a clear indication of $\mathrm{CR}$ origin;

c) contrary to $\mathrm{Li}$, but similarly to $\mathrm{Be}$, it does not show any plateau value at low metallicity, i.e. no indication for a primordial origin;

d) differences in the boron (and beryllium) content between stars otherwise very similar have been detected.

Figures 2 and 3 represent some of these points: they show respectively how $\mathrm{B}_{N L T E}$ and the $\mathrm{B}_{N L T E} / \mathrm{Be}$ ratio behave with metallicity. All the data plotted were derived from the resonant doublet at $2500 \AA$, making use of the latest $\mathrm{Ku}$ rucz model atmospheres (with the "approximate overshooting" option switched off) and his ATLAS and SYNTHE codes (Kurucz 1993). Details on this part of the analysis can be found in Primas et al. (1999). The $\chi^{2}$-fit computed by Duncan et al. (1997) is superimposed just for comparison (it has a slope of 0.7). Most of the few points (filled circles) falling below the fit are slightly evolved stars for which the lower content of boron can be easily explained via stellar destruction and/or mixing (cf Primas et al. 1999). The only exception is the point at $[\mathrm{Fe} / \mathrm{H}]=-1.80$ for which the low boron content may be intrinsic (cf Primas et al. 1998). The two questionmarks have the purpouse of reminding the reader that the detection of boron in these two very metal-deficient stars is under debate (cf García-López et al. 1998). The open triangle marked with "u.l." is an upper limit.

The first observable (point a) above) is difficult to interpret in the classical CR spallation scenario, in which B is a secondary element: a slope of 2 is in fact expected in the logarithmic plane B vs Fe, since supernovae are the primary source for both cosmic rays and $\mathrm{O}$ nuclei, and then in a separate process cosmic rays and $\mathrm{O}$ create $\mathrm{B}$ (and $\mathrm{Be}$ ). Besides, adopting $\mathrm{CR}$ spectra similar to those observed in near-Earth space, this class of models underproduce the isotopic ratio ${ }^{11} \mathrm{~B} /{ }^{10} \mathrm{~B}$ observed in meteorites to be $\sim 4$ (cf Chaussidon \& Robert 1994).

While the plateau showing near-uniformly lithium abundances in sufficiently warm halo stars points to a cosmological (non-galactic) origin for a substantial fraction of the observed lithium, the correlation of $\mathrm{B}$ (and $\mathrm{Be}$ ) with metallicty in halo stars requires these elements to be made in the early Galaxy. Any primordial component must be lower than the abundances derived from current observations and would manifest itself as a plateau in the low-metallicity limit.

A first remark to the observed linearity was that the net rate of boron production does not seem to depend on the CNO abundances in the ISM. This led to the suggestion that the CR spallation most important for light elements production could be $\mathrm{C}$ and $\mathrm{O}$ nuclei colliding with ambient protons and $\alpha$-particles (i.e., a "reverse" spallation reaction, which may decouple light element production from the metallicity of the ISM), probably in regions of massive star formation. Although able to reproduce the observed linearity, later calculations showed that it underproduces the $\mathrm{LiBeB}$ yields by at least one order of magnitude (e.g. Parizot \& Drury 1999). 


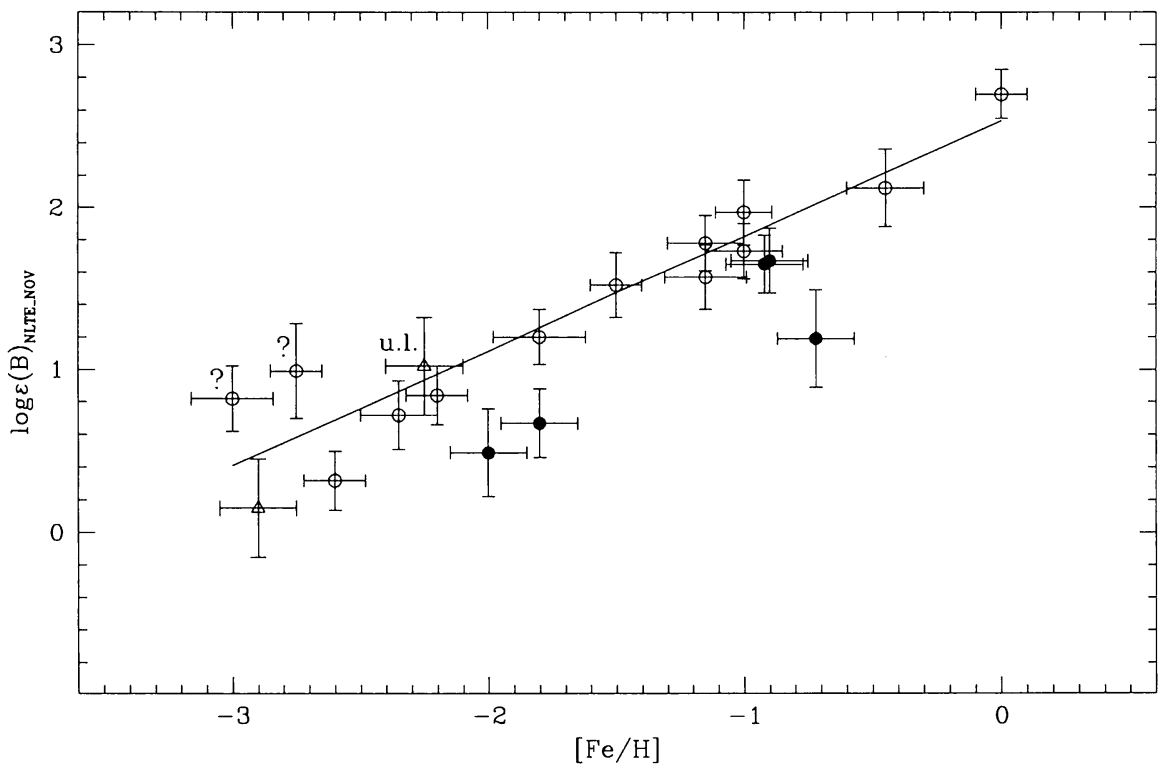

Figure 2. NLTE B abundances versus metallicity. The data points were taken from analyses of Primas et al. (1999, filled and open circles) and García-López et al. (1998, triangles). The data point at solar metallicity is the recent determination of photospheric solar boron by Cunha \& Smith (1999).

It is probably safe to say that observations of $\mathrm{B}$ in low metallicity halo stars formed during the first $10^{9}$ years of galactic evolution show that cosmic ray acceleration must have taken place in the early Galaxy. The $\mathrm{B} / \mathrm{Be}$ ratio observed to be almost constant at all metallicities represents a strong evidence for CR spallation (cf Figure 3). The observed abundances of B relative to Fe, which in the early Galaxy is almost exclusively produced in Type II supernovae $(\mathrm{SNe})$, strongly suggest that the cosmic ray acceleration is also related to such $\mathrm{SNe}$ with the particles being accelerated out of freshly nucleosynthesized matter before it mixes into the ambient, essentially non-metallic ISM. Unfortunately, the current data are not sufficient yet to further constrain the proposed scenarios. The latest developments, nicely reviewed at this meeting by several investigators (cf Beers, Cassé, Olive, Parizot, Ramaty, this volume) seem to explore two main scenarios: $\mathrm{LiBeB}$ production in superbubbles induced by the collective effect of $\mathrm{SNe}$ in $\mathrm{OB}$ associations and a two-component source of GCR in a SN-driven chemical evolution model of the Galactic halo in which both interstellar gas and fresh SN ejecta trapped in the shell undergo SN shock wave acceleration. 


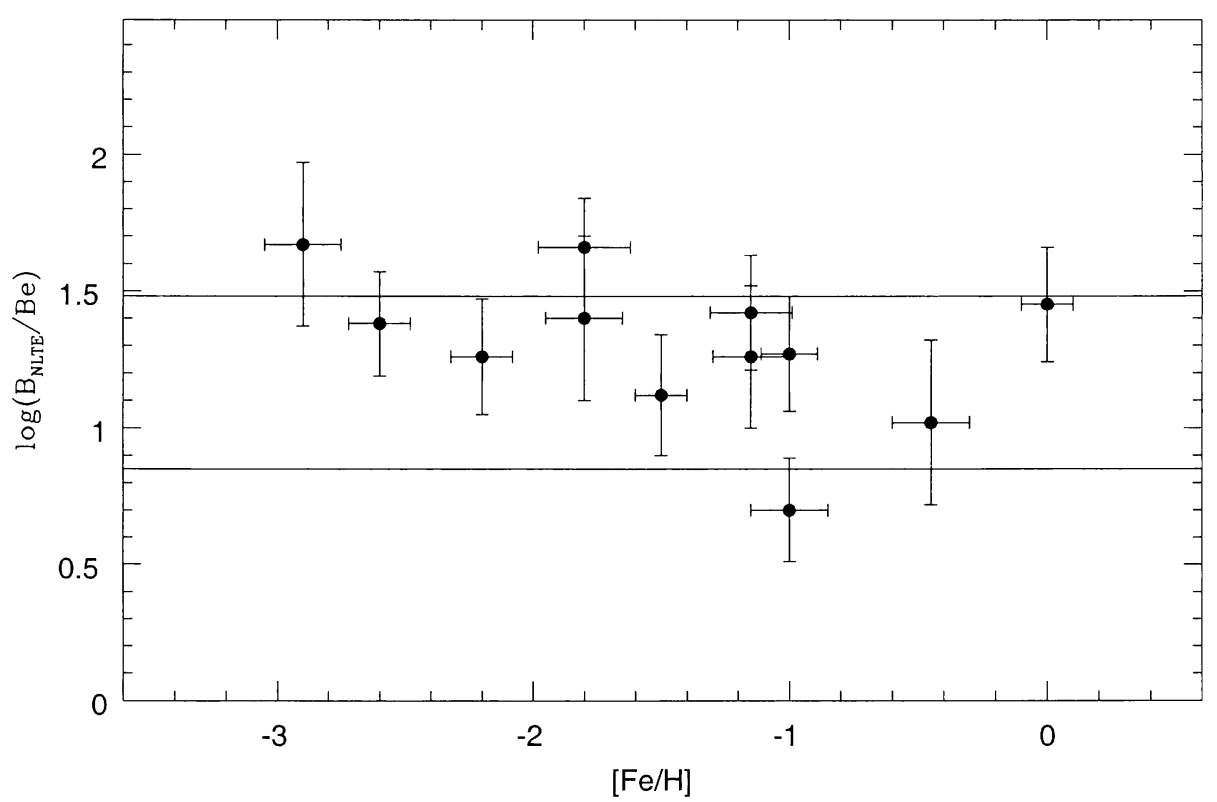

Figure 3. $\quad \mathrm{B}_{N L T E} / \mathrm{Be}$ versus metallicity for the same sample of stars displayed in Fig. 2, but restricting the sample to unevolved stars only.

However, despite all these attempts, the observed linearity still defies one simple theoretical explanation. The situation gets even more complicated for boron (compared to beryllium) because a significant contribution to ${ }^{11} \mathrm{~B}$ from neutrino-spallation is very likely (and needed in order to match the observed meteoritic isotopic ratio). Thus, there are at least two main (probably complementary) mechanisms that need to be understood and disentangled.

\section{Summary and Future Prospects}

The abundance of $\mathrm{B}$ has now been measured in halo stars of metallicity as low as $[\mathrm{Fe} / \mathrm{H}]=-3.0$, showing that $\mathrm{B} / \mathrm{Fe}$ is independent of metallicity: $\mathrm{B}$ follows $\mathrm{Fe}$ in direct proportion from the earliest times to the present, with little change of slope (if any) between halo and disk metallicities.

It is clear and well established that the cosmic ray spallation theory as originally proposed in the early 70 s is unable to reproduce the observed trend. Such finding has triggered a re-analysis of this scenario in which B (and Be) are considered to be secondary elements, mostly formed by spallation reactions of galactic cosmic rays impinging on the interstellar medium. Unfortunately, although the trend of the $\mathrm{B} / \mathrm{Be}$ ratio with metallicity suggests that cosmic ray spallation is indeed part of the game, the current data are not sufficient yet to 
discern among the several theoretical solutions that have been developed in the last few years. The differences in the assumptions are often too subtle to be thoroughly tested with the data sample currently available.

However, among the many aspects that still need to be explored in order to improve and narrow the constraints, there is plenty of room for progress. From the analytical point of view, as mentioned earlier, the availability of 3-D hydrodynamical model atmospheres in the near future and the constant improvements in the atomic physics of near-UV transitions will offer us a much more realistic description of the physics, dynamics and composition of stellar atmospheres.

A deeper knowledge of how $[\mathrm{O} / \mathrm{Fe}]$ correlates with metallicity is also very important. The most direct way to interpret boron abundances is indeed to plot $\mathrm{B}$ (as well as $\mathrm{Be}$ ) versus $\mathrm{O}$, instead of $\mathrm{Fe}$, because oxygen is directly involved in the same cosmic-ray spallation reactions responsible for the production of boron. Fields \& Olive (1999) have shown that if the newly found [O/Fe] trend versus $[\mathrm{Fe} / \mathrm{H}]$ is adopted (i.e. an increasing oxygen abundance as metallicity decreases, e.g., Israelian, García-López, \& Rebolo 1999), then the observed linearity may still be reproduced within the context of the classical theory of cosmic-ray spallation. However, the determination of oxygen abundances is a very delicate matter and suffers from several uncertainties; therefore, until these high oxygen values in the most metal-deficient stars are further confirmed (ideally by using a larger selection of oxygen abundance indicators), any comparison between $\mathrm{B}$ and $\mathrm{O}$ can not be conclusive.

As far as observational efforts are concerned, there is no doubt that enlarging the sample of available data represents the next essential step, especially at the lowest metallicity end. The detection of boron in 2 out of the 3 most metal-poor objects ever observed with HST is still under debate (cf discussion of Fig. 2 in Section 3), hence few more data points around $[\mathrm{Fe} / \mathrm{H}] \sim-3.0$ would be of invaluable help. Unfortunately, one of the biggest unknown here is the timescale on which such a progress will be realistically achieved for boron, whereas beryllium observations have indeed the big advantage of being observable from ground. The potentialities of the new Ultraviolet and Visible Echelle Spectrograph mounted on the ESO VLT Kueyen Telescope revealed during the period of first commissioning suggest that UVES (available to the scientific community starting April 1, 2000) will have a strong impact on this specific field of research.

\section{References}

Asplund, M., Nordlund, A., Trampedach, R., \& Stein, R. 1999, A\&A, 346L, 17 Asplund, M. 1999, this volume

Beers, T. C. 2000, this volume

Boesgaard, A. M., \& Heacox, W. D. 1978, ApJ, 226, 888

Boesgaard, A. M., Deliyannis, C. P., King, J. R., Ryan, S. G., Vogt, S. S., Beers, T. C. 1999 AJ, 117, 1549

Cassé, M. 1999, this volume

Chaussidon, M., \& Robert, F. 1994, Meteoritics, 29, 455 
Cunha, K., Lambert, D. L., Lemke, M., Gies, D. R., \& Roberts, L. C. 1997, ApJ, 478, 211

Cunha, K., \& Smith, V. V. 1999, ApJ, 512, 1006

Duncan, D.K., Lambert, D. L., \& Lemke, M. 1992, ApJ, 401, 584

Duncan, D. K., Primas, F., Rebull, L. M., Boesgaard, A. M., Delyiannis, C. P., Hobbs, L. M., King, J. R., \& Ryan, S. G. 1997, ApJ, 488, 338

Edvardsson, B., Gustafsson, B., Johansson, S., G., Kiselman, D., Lambert, D. L., Nissen, P. E., \& Gilmore, G. 1994, A\&A, 290, 176

Fields, B. D., \& Olive, K. A. 1999, ApJ, 516, 797

García-López, R., Lambert, D. L., Edvardsson, B., Gustafsson, B., Kiselman, D., \& Rebolo, R. 1998, ApJ, 500, 241

Israelian, G., García-López, R. J., \& Rebolo, R. 1998, ApJ, 507, 805

Johansson, S. G., Litzen, U., Kasten, J., \& Kock, M. 1993, ApJ, 403L, 25

Kiselman, D., \& Carlsson, M. 1996, A\&A, 311, 680

Kohl, J. L., Parkinson, W. H., \& Withbroe, G. L. 1977, ApJ, 212L, 101

Kurucz, R. L., 1993, CD-ROM \# 1, 13, 18

Lambert, D. L., Sheffer, Y., Federman, S. R., Cardelli, J. A., Sofia, U. J, \& Knauth, D. C. 1998, ApJ, 494, 614

Meléndez, J., \& Barbuy, B. 2000, this volume

Molaro, P. 1987, A\&A, 183, 241

Nicholson, S. B., \& Perrakis, N. 1928, ApJ, 68, 327

Olive, K. 2000, this volume

Orito, M., Kajino, T., Boyd, R. N., \& Mathews, G. J. 1997, ApJ, 488, 515

Parizot, E., \& Drury, L. 1999, A\&A, 346, 686

Parizot, E. 2000, this volume

Peterson, R. C., Carney, B. W., \& Smith, H. 1999, ApJ, submitted

Praderie, F., Milliard, B., Pitois, M. L., \& Boesgaard, A. M. 1977, ApJ, 214, 130

Primas, F., Duncan, D. K., \& Thorburn, J. A. 1998, ApJ, 506L, 51

Primas, F., Duncan, D. K., Peterson, R. C., \& Thorburn, J. A. 1999, A\&A, 343, 545

Ramaty, R. 2000, this volume

Rebull, L. M., Duncan, D. K., Johansson, S. G., Thorburn, J. A., \& Fields, B. D. 1998, ApJ, 507, 387

Reeves, H., Fowler, W. A., \& Hoyle, F. 1970, nature, 226, 727

Rowland, H. A., \& Tatnall, R. R. 1895, ApJ, 1, 14

Spite, F., \& Spite, M. 1982, A\&A, 115, 357

Venn, K. A., Lambert, D. L., \& Lemke, M. 1996, A\&A, 307, 849 